\title{
EVALUATION OF INFLAMMATION AND ENDOTHELIAL DYSFUNCTION BIOMARKERS IN CHRONIC KIDNEY DISEASE (CKD) PATIENTS IN SOKOTO, NIGERIA
}

\author{
${ }^{* 1}$ Otitolaiye, C. A., ${ }^{2}$ Sahabi, D. M., ${ }^{3}$ Makusidi, A. M., ${ }^{2}$ Saidu, Y. and ${ }^{2}$ Bilbis, L. S.
}

\author{
${ }^{* 1}$ Department of Biochemistry, Sokoto State University, Sokoto, Nigeria. \\ Phone: 08067301981 Email: catherineotits@yahoo.com \\ ${ }^{2}$ Department of Biochemistry, Usmanu Danfodiyo University, Sokoto, Nigeria \\ Phone: 08036131987 Email: yusdab@yahoo.com \\ ${ }^{3}$ Department of Medicine, Usmanu Danfodiyo University Teaching Hospital, Sokoto, Nigeria \\ Phone: 08036057382 Email: makusidi1@yahoo.com
}

\begin{abstract}
Inflammation and endothelial dysfunction have been known to be involved in the pathogenesis of cardiovascular diseases. As such, examining the levels of inflammation and endothelial dysfunction is very critical to the prevention of cardiovascular diseases among chronic kidney disease (CKD) patients. This study aimed to investigate the progression of inflammation and endothelial dysfunction among CKD patients in Sokoto. A total of $67 \mathrm{CKD}$ patients were divided into 5 groups based on the stages of their kidney disease calculated using the MDRD 4-variable equation for estimated glomerular filtration rate (eGFR). The presence of inflammation was determined by C-Reactive Protein (CRP) and Tumor Necrosis Factor alpha $(\mathrm{TNF}-\alpha)$, while endothelial dysfunction was determined by the levels of Asymmetric dimethylarginine (ADMA) using ELISA kits. The mean eGFR of the patients was $49.97 \pm 4.69 \mathrm{ml} / \mathrm{min} / 1.73 \mathrm{~m}^{2}$. There was significant increase $(\mathrm{p}<0.05)$ in CRP, TNF- $\alpha$ and ADMA of the CKD patients across the stages as compared to the non-CKD subjects. It was observed that as the CRP, TNF- $\alpha$ and ADMA increase, the eGFR significantly $(\mathrm{p}<0.05)$ decreases. Both CRP and TNF- $\alpha$ indicated a significantly positive correlation $(\mathrm{p}<0.05)$ with ADMA. The results indicated progressive increase in inflammation and endothelial dysfunction as CKD deteriorates. In addition, increased levels of inflammation could directly affect endothelial dysfunction, thereby aggravating cardiovascular morbidity and mortality among CKD patients in Sokoto.
\end{abstract}

Keywords: cardiovascular disease, chronic kidney disease, endothelial dysfunction, glomerular filtration rate, inflammation

LICENSE: This work by Open Journals Nigeria is licensed and published under the Creative Commons Attribution License 4.0 International License, which permits unrestricted use, distribution, and reproduction in any medium, provided this article is duly cited.

COPYRIGHT: The Author(s) completely retain the copyright of this published article.

OPEN ACCESS: The Author(s) approves that this article remains permanently online in the open access (OA) mode.

QA: This Article is published in line with "COPE (Committee on Publication Ethics) and PIE (Publication Integrity \& Ethics)". 


\section{INTRODUCTION}

Cardiovascular disease (CVD), defined as the cluster of hypertensions, dyslipidemia, atherosclerosis, myocardial infarction, angina pectoris and heart failure, are the leading cause of mortality, both in the general population and in patients with CKD (Port, 1994). Cardiovascular disease (CVD) occurs even at the earliest stages of CKD (DiAngelantonio et al., 2010) and majority of CKD patients in stages 3 to 5 usually die of cardiovascular complication rather than the CKD progression (Alebiosu et al., 2006; USRDS, 1998). Many risk factors, traditional and nontraditional, are involved in the development of CVD and progression in CKD patients. The traditional risk factors include diabetes, hypertension, high cholesterol, obesity, lack of exercise, smoking, increased age, and family history (Ardhanari et al., 2014), while the non-traditional risk factors are homocysteinemia, anemia, inflammation, endothelial dysfunction, proteinuria and oxidative stress. In patients with progressive CKD, the issue is further complicated because of the appearance of uremia-specific risk factors with the potential of contributing to endothelial and vascular dysfunction in the patients (Ardhanari et al., 2014; Amaresan, 2005).

Inflammation is fundamentally a protective response whose ultimate goal is to rid an individual of both the initial cause of cell injury (e.g. microbes or toxins), clear out necrotic cells and tissues damaged from the inflammatory process and to initiate tissue repair. Inflammatory process ceases when the mechanism responsible for cellular intermediates are inhibited and healing takes place (Sprague and Khalil, 2009).

However, CKD patients may experience different kinds of metabolic changes due to kidney disease which might be responsible for increase in inflammatory mediators such as cytokines. These and other inflammatory mediators such as activated $\mathrm{T}$ cells and mast cells then attach themselves to the endothelium thereby causing damage (Shrivastava et al., 2015). Damage to the endothelial wall triggers a cascade of events that modulates inflammatory response, leading to recruitment of white blood cells into the blood vessel wall, where they give rise to abnormal foam cells and initiate the development of atherosclerotic lesions (Shrivastava et al., 2015). As such normal endothelial function is very critical to the prevention of vascular diseases, including atherosclerosis.

This study therefore aims to investigate the progression of inflammation and endothelial dysfunction among CKD patients in Sokoto, by estimating TNF- $\alpha$, CRP and ADMA which have been proposed as reliable makers of inflammation and endothelial dysfunction.

\section{MATERIALS AND METHOD}

This study was approved by the Ethics Committee of Usmanu Danfodiyo University Teaching Hospital Sokoto. The participants signed the free and informed consent forms. A total of 67 patients were recruited from both Usmanu Danfodiyo University and Sokoto Specialist Hospital, Sokoto. The inclusion criteria involved all patients with the various stages of $\mathrm{CKD}$ present in the clinics and the exclusion criteria were patients not willing to participate and patients with established sepsis. The CKD patients were divided into 5 groups based on the severity of their kidney disease calculated using estimated glomerular filtration rate (eGFR).

The concentration of creatinine was determined using colorimetric method reported by Allen (1982). An ELISA kit from CalBiotech (USA) was used for the assay of C-reactive protein (Kindmark, 1972). ELISA kits from Biotain Pharma Co. China were used for the assay of TNF- $\alpha$ and ADMA according to the manufacturer's instruction. 


\section{STATISTICAL ANALYSIS}

The results were expressed as Means \pm SEM. Student's t-test was used to compare the healthy control and the CKD patients. ANOVA was used to compare between the different stages and with the control with Dunnet post test. Pearson's correlation was used to determine the relations between the different variables. $\mathrm{P}<0.05$ was considered as statistically significant. Statistics was by Graphpad instat3 version 3.02, USA.

\section{RESULTS}

Table 1 summaries the clinical investigations of the CKD patients and healthy controls. Out of the 67 CKD patients, $36(53.7 \%)$ were males while $31(46.3 \%)$ were females. The age is not statistically different between the groups, with the mean age of both male and female CKD patients as $47.17 \pm 1.77$ years ranging from 21 to 75 years. The mean plasma creatinine level of the patients was significantly increased $(\mathrm{p}<0.05)$ and consequently, the eGFR of the patients was significantly decreased $(\mathrm{p}<0.05)$ in comparison to the healthy control.

Table 1: Demographic Characteristics of CKD Patients and Healthy Control Subjects in Sokoto

\begin{tabular}{|c|c|c|c|}
\hline Parameters & CKD Patients $(\mathrm{n}=67)$ & Control $(\mathrm{n}=15)$ & P-value \\
\hline $\operatorname{Sex}(M: F)$ & $36: 31$ & $7: 8$ & \\
\hline Age (yrs) & $47.47 \pm 1.77$ & $48.13 \pm 5.26$ & 0.9067 \\
\hline Creatinine $(\mathrm{mg} / \mathrm{dl})$ & $3.55 \pm 0.49$ & $0.79 \pm 0.05$ & 0.0001 \\
\hline $\mathrm{eGFR}\left(\mathrm{ml} / \mathrm{min} / 1.73 \mathrm{~m}^{2}\right)$ & $49.97 \pm 4.69$ & $117.53 \pm 7.99$ & 0.0001 \\
\hline
\end{tabular}

Values are mean \pm standard error of means. eGFR $=$ Estimated Glomerular Filtration Rate

The results of the biomarkers of inflammation and endothelial dysfunction in the CKD patients as compared to apparently healthy control subjects were listed in Table 2. Patients with CKD had significantly increased (p < 0.05) levels of TNF- $\alpha$, CRP and ADMA as compared to the healthy control subject.

Table 2: Inflammation and Endothelial Dysfunction Markers in CKD Patients in Sokoto

\begin{tabular}{llll} 
Parameters & CKD Patients $(\mathrm{n}=67)$ & Control $(\mathrm{n}=15)$ & P-Value \\
\hline TNF- $\alpha(\mathrm{pg} / \mathrm{ml})$ & $10.34 \pm 0.67$ & $6.01 \pm 0.23$ & 0.0001 \\
CRP $(\mathrm{mg} / \mathrm{l})$ & $17.88 \pm 0.77$ & $7.27 \pm 0.80$ & 0.0001 \\
ADMA $(\mu \mathrm{mol} / \mathrm{l})$ & $0.15 \pm 0.01$ & $0.08 \pm 0.02$ & 0.0036 \\
\hline
\end{tabular}

Values are mean \pm standard error of means. TNF- $\alpha=$ Tumor Necrosis Factor alpha. $\mathrm{CRP}=\mathrm{C}-$ reactive protein. $\mathrm{ADMA}=$ Asymmetric Dimethylarginine . 
The results of the biomarkers of inflammation at different stages of CKD are presented in Fig 1 below. There was a significantly steady increase $(\mathrm{p}<0.05)$ in CRP of the CKD subjects across the stages and when compared to the healthy control subjects

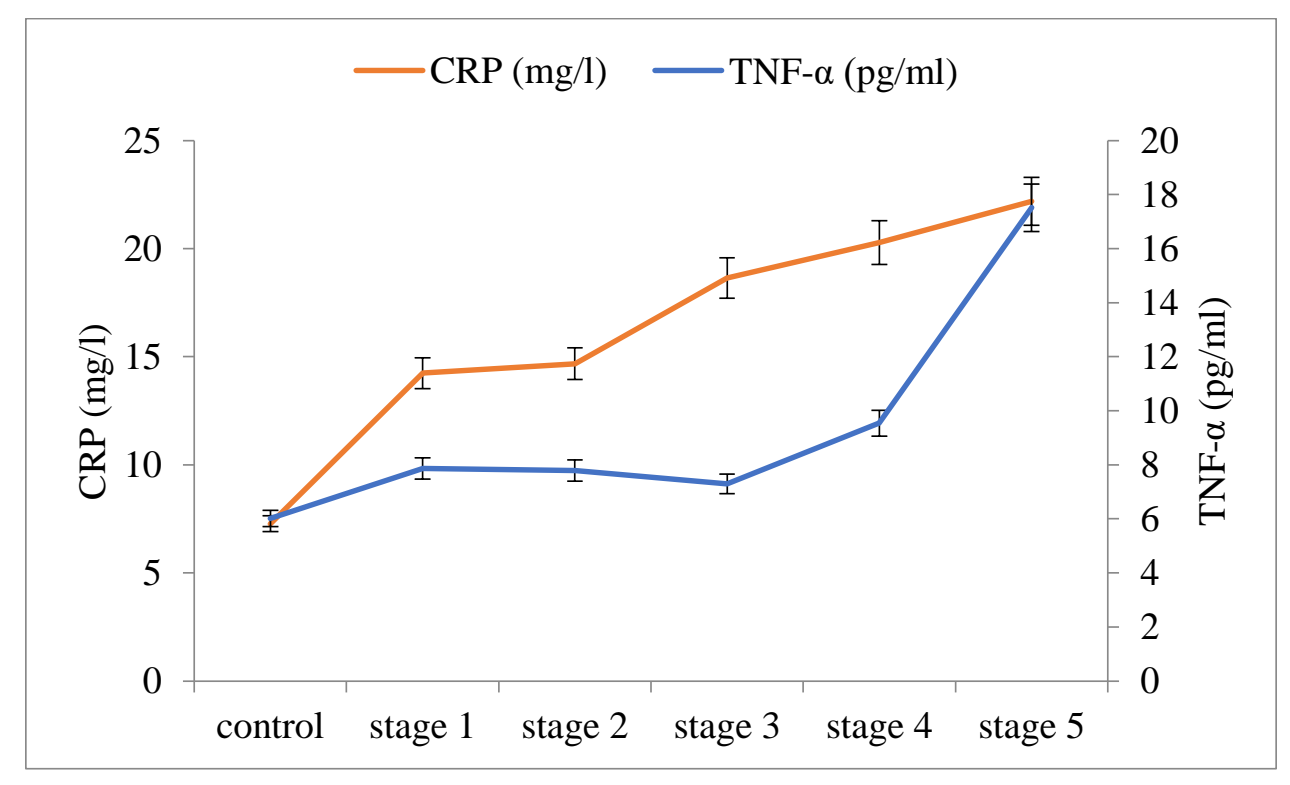

Figure 1: Plasma levels of TNF- $\alpha(\mathrm{pg} / \mathrm{ml})$ and CRP $(\mathrm{mg} / \mathrm{l})$ at Various Stages of CKD

The result of the biomarker of endothelial dysfunction at various stages of CKD is presented in Fig 2. There was a significant $(\mathrm{p}<0.05)$ increase in ADMA from stage 1 to stage 5 of the disease.

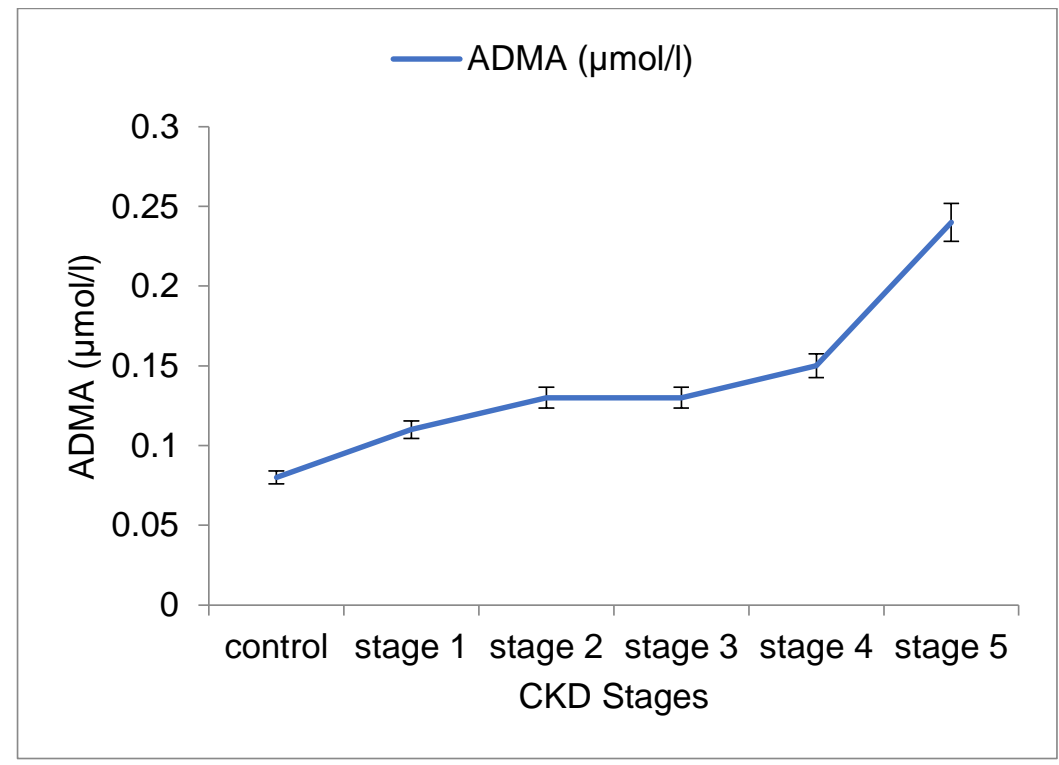

Figure 2: Plasma Level of ADMA at Various Stages of CKD 
Table 3 shows a significant negative correlation between eGFR and the cardiovascular risk factors (TNF- $\alpha$, CRP and ADMA). It was observed that the cardiovascular factors increase as the kidney function deteriorates.

Table 3: Correlation Coefficients of eGFR with Cardiovascular Risk Factors in CKD Patients in Sokoto.

\begin{tabular}{lcc}
\hline Parameters & \multicolumn{2}{c}{} \\
\cline { 2 - 3 } & r-value & P- value \\
\hline Tumor Necrosis Factor- $\alpha(\mathrm{pg} / \mathrm{ml})$ & -0.520 & 0.0001 \\
C-Reactive Protein $(\mathrm{mg} / \mathrm{l})$ & -0.487 & 0.0001 \\
Asymmetric Dimethylarginine $(\mu \mathrm{mol} / \mathrm{l})$ & -0.348 & 0.0039 \\
\hline
\end{tabular}

The correlation of ADMA with markers of inflammation (TNF- $\alpha$, CRP) indicated a significant $(\mathrm{p}<0.05)$ positive correlation coefficient as shown in the figures below (Fig 3 and 4).

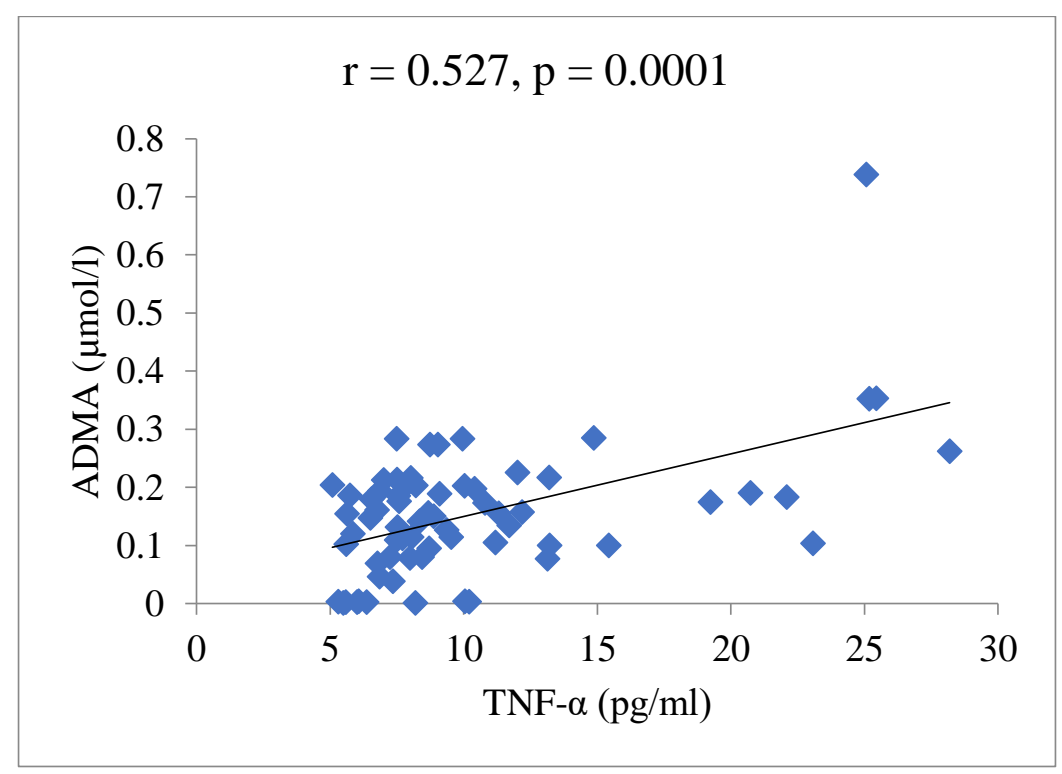

Figure 3: Correlation Graph of TNF- $\alpha$ and ADMA Levels in CKD Patient 


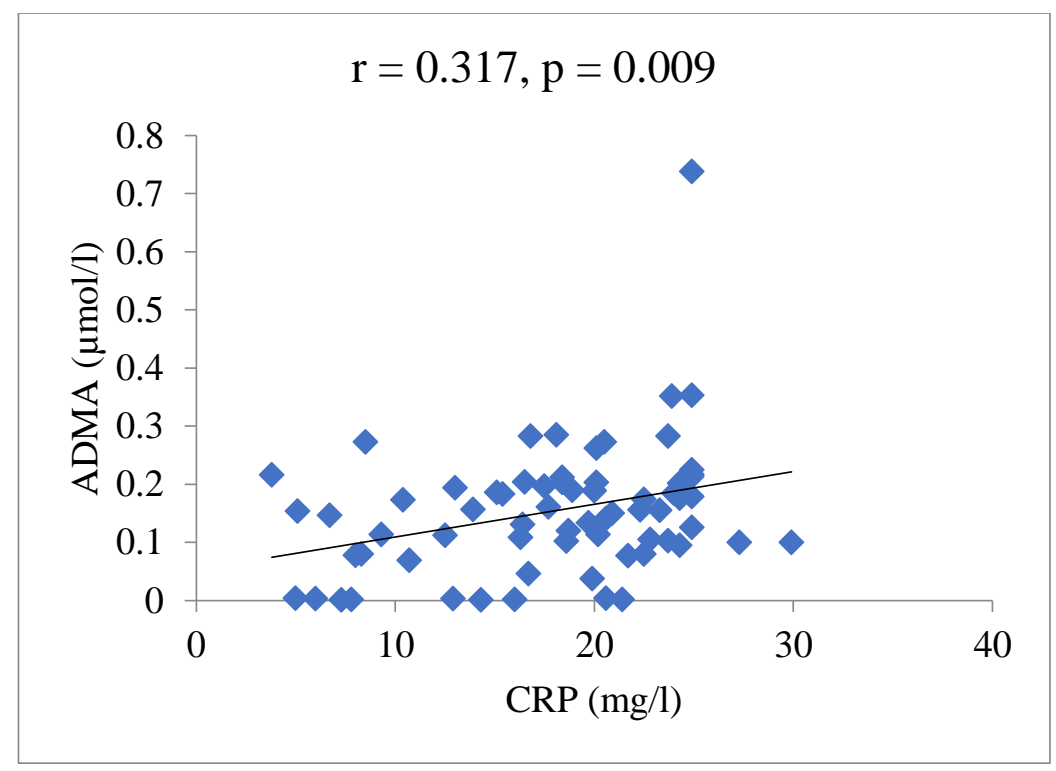

Figure 4: Correlation Graph of CRP and ADMA Levels in CKD Patients

\section{DISCUSSION}

Inflammation and endothelial dysfunction both play a crucial role in the development of cardiovascular disease. As a result, normal levels of inflammatory markers as well as endothelial dysfunction markers are vital to the prevention of cardiovascular disease among CKD patients. This study therefore aims to investigate the progression of inflammation and endothelial dysfunction among CKD patients in Sokoto.

In this study, both TNF- $\alpha$ and CRP were found to be significantly elevated in the CKD patients as compared to the healthy controls (Table 2). A steady increase in inflammatory markers (TNF- $\alpha$ and CRP) across the CKD stages were also observed in this study (Fig 1). Previous studies have shown that CRP enhances cardiovascular risk (Zimmermann et al., 1999) predicts mortality in hemodialysis (Bergstrom, 1995), as well as in peritoneal dialysis patients. The highest increase in the inflammatory biomarkers was observed in stage 5 of the CKD. This could be due to the dialysis procedure and the components of the dialysate. Among the numerous effects of TNF- $\alpha$ are the upregulation of cytokines such as IL-6 and stimulation of acute phase reactants, CRP (Sprague and Khalil, 2009). CRP in turn, have been found to directly contribute to the early phase of atherosclerosis by deposition on the intima, which precedes the appearance of monocytes (Torzewski and Lackner, 2006). In addition, previous studies have shown that CRP could reduce nitric oxide production, stimulates endothelin-1 formation, and induces some of the steps involved in atherosclerosis process (Park and Park, 2015; Labarrere and Zaloga, 2004). Also, in this study a significant negative correlation was observed between TNF- $\alpha$ and eGFR (Table 3) as well as between CRP and eGFR (Table 3). This implies that as the TNF- $\alpha$ and CRP increases, the kidney function deteriorates. It is therefore possible that the increasing inflammation could result in more damaging effect on the kidney.

In addition, this study showed the level of ADMA increased across the stages from stage 1 to stage 5 (Fig 2). According to the findings of Yilmaz et al., (2006) and Kocak et al. (2008), ADMA, of which levels were elevated in CKD interferes with NO generation, through the competitive inhibition of the NO-synthase enzyme, 
by decreasing NO availability and therefore impair endothelial function (Yilmaz et al., 2006; Kocak et al. 2008). Nitric oxide (NO), a potent endogenous vasodilator, inhibits the aggregation of platelets, inhibits the adhesion of monocytes and leukocytes to the endothelium, attenuates smooth muscle cell proliferation and inhibits the oxidation of LDL (Dessy and Ferron, 2004; Bian et al., 2008). With advancing renal failure and loss of renal mass, ADMA accumulates leading to consequent endothelial dysfunction which in turn may lead to arterioslerotic remodeling of vascular beds and to a large extent is responsible for the increased cardiovascular morbidity and mortality in patients with CKD (Kaysen and Eiserich, 2004; Galle et al., 2003).

Damage to endothelial walls through the accumulation of ADMA could trigger a cascade of events that modulates inflammatory response, leading to the recruitment of white blood cells into the blood vessel wall, where they give rise to abnormal foam cells and initiate the development of atherosclerotic lesions (Shrivastava et al., 2015).

A highly significant positive correlation was observed between TNF- $\alpha$, CRP and ADMA (Fig 3 and Fig 4). These suggest increased inflammation could promote endothelial dysfunction among the CKD patients thereby increasing their risk for endothelial damage. According to Blake and Ridker (2001), inflammatory cytokines could induce endothelial cells and leukocytes to express adhesion molecules, such as vascular cell adhesion molecules (VCAM) and intercellular adhesion molecules (ICAM), monocyte chemotactic protein-1, E-selectin, P-selectin, and interleukin-6, resulting in a worsening of endothelial cell injury (Blake and Ridker, 2001). If perpetuated long enough, dysfunction of endothelial cells is followed by their apoptosis, which can finally result in functional and structural disintegration of the endothelial cell layer and consequent endothelial damage (Park and Park, 2015; Hansson, 2005). This process leads to progressive atherosclerotic disease in larger vessels and/or complete disruption of smaller (tissue) blood vessels and consequent cardiovascular disease in the CKD patients.

\section{CONCLUSION}

In conclusion, TNF- $\alpha$ and CRP (markers of inflammation) and ADMA (endothelial dysfunction marker) significantly increased among the CKD patients as the kidney function deteriorates, thereby aggravating cardiovascular morbidity and mortality among the CKD patients in Sokoto.

\section{REFERENCES}

Amaresan, M.S. (2005). Cardiovascular disease in chronic kidney disease. Indian Journal of Nephrology 15: 1-7

Allen, L. K. (1982). Creatinine determination. Clinical Chemistry. 28:555.

Alebiosu, C. O., Ayodele, O. O., Abbas, A. and Olutoyin A. I. (2006) Chronic renal failure at the Olabisi Onabanjo Teaching Hospital, Sagamu, Nigeria. African Health Science 6:132-8.

Ardhanari, S., Alpert, M.A. and Aggarwal, K. (2014) Cardiovascular disease in CKD: Risk factors, Pathogenesis, and Prevention. Advances in Peritoneal dialysis. Vol 30: 40-53

Bergstrom, J. (1995). Why are dialysis patients malnourished? American Journal of Kidney Disease. 26(1): 229241

Bian, K., Doursout, M.F., Murad, F. (2008) Vascular system: Role of nitric oxide in cardiovascular diseases. The Journal of Clinical Hypertension. 10(4): 304-10 
Blake, G.J. and Ridker, P.M. (2001) Novel clinical markers of vascular wall inflammation. Circulation Research 89: 763-771

Dessy, C. and Ferron, O. (2004). Pathophysiological roles of nitric oxide: In the heart and the coronary vasculature. Current Medical Chemistry-Anti-inflammatory and anti-allergy agents in medicinal chemistry. 3(3):207-216

Di-Angelantonio, E., Chowdhur,y R., Sarwar, N., Aspelund, T., Danesh, J. and Gudnason, V. (2010) Chronic kidney disease and risk of major cardiovascular disease and non-vascular mortality: prospective population-based cohort study. British Medical Journal. 341:c4986

Galle, J., Quaschning, T., Seibold, S., Wanner C. (2003). Endothelial dysfunction and inflammation. What is the link? Kidney International Supplementary 84:545-549

Hansson, G. K. (2005). Inflammation, atherosclerosis, and coronary artery disease. New England Journal of Medicine 352: 1685-1695.

Kaysen, G. and Eiserich, J. (2004). The role of oxidative stress-altered lipoprotein structure and function and microinflammation on cardiovascular risk in patients with minor renal dysfunction. Journal of the American Society of Nephrology 15: 538-548

Kindmark, C.O. (1972). The Concentration of C-reactive protein in Sera from Healthy Individuals. Scandinavian Journal of Clinical Laboratory Investigation 29: 407-411

Kocak, H., Gumulu, S., Ermis, C., Mahsereci, E., Sahin, E., Gocmen, A.Y. (2008) Oxidative stress and asymmetric dimethylarginine is independently associated with carotid intima media thickness in peritoneal dialysis patients. American Journal of Nephrology 28: 91-96

Labarrere, C.A. and Zaloga, G.P. (2004). C-reactive protein: from innocent bystander to pivotal mediator of atherosclerosis. American Journal of Medicine. 117: 499-507.

Park, K-H and Park, W.J. (2015). Endothelial dysfunction: Clinical implications in cardiovascular and therapeutic approaches. Journal of Korean Medical Science. 30(9): 1213-1225

Port, F.K. (1994) Morbidity and mortality in dialysis patients. Kidney International 46: 1728-1737

Sprague, A.H. and Khalil, R.A. (2009) Inflammatory cytokines in vascular dysfunction and vascular disease. Biochemical Pharmocology 78(6): 539-552

Shrivastava, A.K., Singh, H.V., Raizada A. and Singh S.K. (2015) C-reactive protein, inflammation and coronary heart disease. The Egyptian Heart Journal 67(2): 89-97

Torzewski, M. and Lackner, K. J. (2006) "Initiation and progression of atherosclerosis — enzymatic or oxidative modification of low-density lipoprotein?” Clinical Chemistry and Laboratory Medicine.44(12):13891394

US Renal Data System, USRDS (1998) Annual data report. American Journal of Kidney Disease. 32(1): S69S80. 
Yilmaz, M. I., Saglam, M., and Saglam, M. (2006) "The determinants of endothelial dysfunction in CKD: oxidative stress and asymmetric dimethylarginine," American Journal of Kidney Diseases. 47(1): 4250

Zimmermann, J., Herrlinger S., Pruy A., Metzger T., Wanner C (1999). Inflammation enhances cardiovascular risk and mortality in hemodialysis patients. Kidney International 55: 648-658 